after vaccination will be assessed. This trial will reveal whether chemotherapy, immunotherapy, or chemo-immunotherapy influences how patients respond to vaccination and could serve as a model for translational studies of other vulnerable populations or comparable cohorts vaccinated with different vaccines against COVID-19.

Understanding whether this group of patients can mount a sufficient immune response to a vaccine against COVID-19 will provide information for supporting and counseling them during this pandemic and will give them a voice. Is the antibody titer high enough to be protective against COVID-19? What is the durability of antibody titers? Do these patients mount a $\mathrm{T}$ cell response sufficient to support the formation of memory B cells? Are two vaccinations sufficient, or are additional doses required? Are alternative measures required?

To assure rapid dissemination of knowledge, we aim to make the results publicly available as soon as possible. Moreover, data sharing will allow comparison between VOICE results and those from other studies for rapid amplification of the knowledge gained.

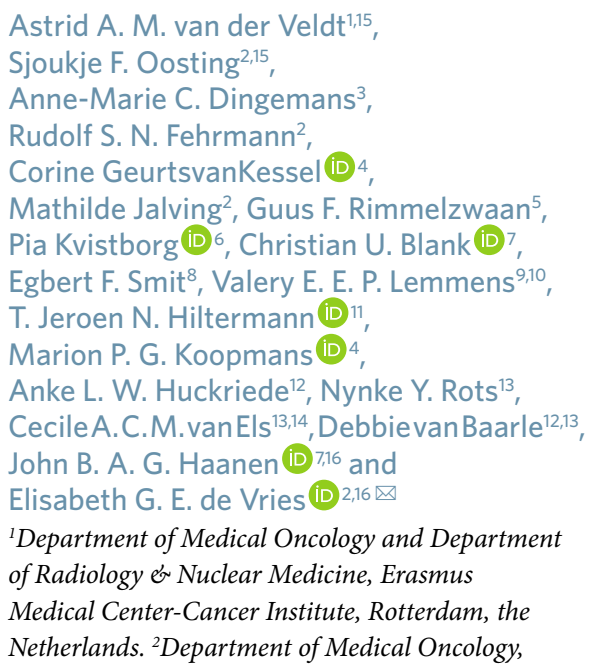

University Medical Center Groningen, University of Groningen, Groningen, the Netherlands. ${ }^{3}$ Department of Respiratory Medicine, Erasmus Medical Center-Cancer Institute, Erasmus Medical Center, Rotterdam, the Netherlands. ${ }^{4}$ Department of Viroscience, Erasmus Medical Center, Rotterdam, the Netherlands. ${ }^{5}$ Research Center for Emerging Infections and Zoonoses, University of Veterinary Medicine Hannover, Hannover, Germany. ${ }^{6}$ Department of Molecular Oncology and Immunology, Netherlands Cancer InstituteAntoni van Leeuwenhoek Hospital, Amsterdam, the Netherlands. ${ }^{7}$ Department of Medical Oncology, Netherlands Cancer Institute-Antoni van Leeuwenhoek Hospital, Amsterdam, the Netherlands. ${ }^{8}$ Department of Thoracic Oncology, Netherlands Cancer Institute-Antoni van Leeuwenhoek Hospital, Amsterdam, the Netherlands. ${ }^{9}$ Comprehensive Cancer Organization the Netherlands/Netherlands Cancer Registry, Utrecht, the Netherlands. ${ }^{10}$ Department of Public Health, Erasmus Medical Center, Rotterdam, the Netherlands. ${ }^{11}$ Department of Pulmonary Diseases, University Medical Center Groningen, University of Groningen, Groningen, the Netherlands. ${ }^{12}$ Department of Medical Microbiology and Infection Prevention, University Medical Center Groningen, University of Groningen, Groningen, the Netherlands. ${ }^{13}$ Center for Infectious Disease Control, National Institute for Public

$\square \quad$ Health and the Environment, Bilthoven, the Netherlands. ${ }^{14}$ Department of Biomolecular Health Sciences, Faculty of Veterinary Medicine, Utrecht University, Utrecht, the Netherlands. ${ }^{15}$ These authors contributed equally: Astrid A. M. van der Veldt, Sjoukje F. Oosting. ${ }^{16}$ These authors jointly supervised this work: John B.A.G. Haanen, Elisabeth G.E. de Vries.

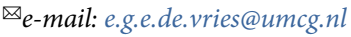

Published online: 15 February 2021

https://doi.org/10.1038/s41591-021-01240-w

\footnotetext{
References

1. Ribas, A. et al. Cancer Discov. 11, 233-236 (2021).

2. $\mathrm{Au}$, L. et al. Cell 183, 4-10 (2020).

3. de Joode, K. et al. Eur. J. Cancer 136, 132-139 (2020).

4. Nederlandse Federatie van Kankerpatiëntenorganisaties. https:// nfk.nl/media/1/Downloads/201210-DJE-corona-II_rapportage _ finaal.pdf (2020).

5. I\&O Research. https://www.ioresearch.nl/actueel/lagere-
} bereidheid-tot-vaccinatie (2020).
6. US Food and Drug Administration. https://www.fda.gov/ emergency-preparedness-and-response/coronavirus-disease2019-covid-19/covid-19-vaccines (accessed January 2021).

7. Garassino, M. C. et al. European Society for Medical Oncology https://www.esmo.org/covid-19-and-cancer/covid-19. vaccination?hit=ehp (2020)

8. American Society of Clinical Oncology. https://www.asco.org/ asco-coronavirus-resources/covid-19-patient-care-information/ covid-19-vaccine-patients-cancer (accessed January 2021).

9. Society for Immunotherapy of Cancer. https://www.sitcancer.org/ aboutsitc/press-releases/2020/sitc-statement-sars-cov-2vaccination-cancer-immunotherapy (2020)

10. Fendler, A. et al. Preprint at https://www.medrxiv.org/content/ $10.1101 / 2020.12 .21 .20248608 \mathrm{v} 1(2020)$

\section{Competing interests}

A.A.M.v.d.V. reports consultancy fees (paid to Erasmus Medical Center) from BMS, MSD, Merck, Sanofi, Eisai, Pfizer, Ipsen, Roche, Pierre Fabre and Novartis. S.F.O. reports research grants from Novartis and Celldex Therapeutics (paid to University Medical Center Groningen). A.-M.C.D. reports consultancy fees (paid to Erasmus Medical Center) from Roche, Eli Lilly, Boehringer Ingelheim, BMS, Amgen, Novartis, Pfizer, Takeda, Chiesi, Pharmamar, Bayer and Sanofi, and research support (paid to Erasmus Medical Center) from BMS, AbbVie and Amgen. Mathilde Jalving reports consultancy fees (paid to University Medical Center Groningen) from BMS, MSD, Merck, Pfizer, AstraZeneca, Pierre Fabre and Novartis. C.U.B. reports an advisory role at BMS, MSD, Roche, Novartis, GSK, AZ, Pfizer, Lilly, GenMab, Pierre Fabre and Third Rock Ventures, research funding from BMS, Novartis and NanoString, and stock ownership in Uniti Cars, and is a co-founder of Immagene BV. E.F.S. reports consultancy fees (all Netherlands Cancer Institute-Antoni van Leeuwenhoek Hospital) from AstraZeneca, Bayer, Bristol Myers Squibb, Daiichi Sankyo, Eli Lilly, Merck, MSD, Novartis, Pfizer, Roche Diagnostics, Roche Genentech and Takeda. T.J.N.H. reports consultancy fees (paid to University Medical Center Groningen) from BMS, MSD, Merck, Boehringer, AstraZeneca and Roche. J.B.A.G.H. reports consultancy roles for Achilles Therapeutics, BioNTech, BMS, GSK, Immunocore, Molecular Partners, MSD, Merck Serono, Neogene Therapeutics, Novartis, Pfizer, Roche/Genentech, Sanofi and Third Rock Ventures, and research grants from Amgen, BMS, BioNTech, MSD, Novartis, Neogene Therapeutics (paid to the Netherlands Cancer Institute-Antoni van Leeuwenhoek Hospital). Elisabeth G.E. de Vries reports an advisory role at Daiichi Sankyo, NSABP and Sanofi (paid to University Medical Center Groningen), and research funding from Amgen, AstraZeneca, Bayer, Chugai Pharma, CytomX Therapeutics, G1 Therapeutics, Genentech, Nordic Nanovector, Radius Health, Regeneron, Roche, Servier and Synthon (paid to University Medical Center Groningen).

\title{
Placebo use and unblinding in COVID-19 vaccine trials: recommendations of a WHO Expert
} Working Group

To the Editor-The grave public-health threat posed by coronavirus disease 2019 (COVID-19) has spurred an unprecedented accelerated approach to vaccine research and deployment. Currently, hundreds of thousands of participants globally are enrolled in COVID-19 vaccine trial research, with more trials imminent or proposed $^{1}$. As of 1 March 2021, multiple 
regulatory agencies ${ }^{2-4}$ and the World Health Organization (WHO $)^{5,6}$ had granted emergency use designation (EUD) to qualifying candidate vaccines against COVID-19, on the basis of early evidence of efficacy and safety. Consequently, qualifying candidate vaccines are being deployed before conclusion of their trials and/or the collection of longer-term data on safety and efficacy.

From a scientific standpoint, it is important for the clinical trials of vaccines granted an EUD to continue to their completion. But the provision of a candidate vaccine under an EUD to millions of people raises urgent questions about the continuation of the control-group arm of these and other trials, and whether trial blinding is still warranted. Current research-ethics guidance documents ${ }^{7-9}$ were not drafted with emergency-use deployment in mind. Given such dilemmas and guidance gaps, the WHO ACT-Accelerator Ethics \& Governance Working Group (ACT: Access to COVID-19 Tools; called 'Working Group' here) has developed a policy brief to guide ethical decision-making in these circumstances: 'Emergency Use Designation of COVID-19 candidate vaccines: Ethical considerations for current and future COVID-19 placebo-controlled vaccine trials and trial unblinding ${ }^{\prime 10}$.

The Working Group has concluded that although there is a scientific imperative to continue trials of vaccines against COVID19 after a candidate vaccine is granted an EUD, there is also an ethical imperative to ensure that trial participants who are at substantial risk of infection with the coronavirus SARS-CoV-2, and severe COVID-19 morbidity or mortality—such as healthcare workers at high to very high risk of acquiring and transmitting the disease, and people above 65 years of age-are in a position to access an EUD vaccine as soon as practically possible, should they wish to do so. Candidate vaccines granted an EUD will probably be deployed in a phased manner to ensure the prioritization of those deemed to be at considerable risk. In settings in which candidate vaccines are introduced under an EUD, investigators should explain the scientific benefit of continued trial participation and the implications of unblinding to trial participants deemed to be at substantial risk of infection, severe morbidity or mortality. Participants should then be offered the opportunity to be unblinded, so that they can make an informed decision about whether to withdraw from the trial and access an EUD vaccine programmatically as soon as practically possible, should they wish to do so. Trial participants who are not deemed to be at substantial risk of SARS-CoV-2 infection and COVID-19 morbidity or mortality and who do not meet prevailing eligibility criteria to access a candidate vaccine granted an EUD should be informed of the scientific benefits of continuing with the trial and should be encouraged to remain enrolled, with full acknowledgment of their right to withdraw from a trial at any point, without penalty. The continued enrollment of as many participants as possible, for as long as possible, will have considerable scientific and public health utility, as doing so will yield invaluable longer-term data on the safety and efficacy of candidate vaccines. Continued enrollment could also highlight the potential risk of emerging variants of SARS-CoV-2 of concern in relation to those previously unexposed to the virus, those previously infected and recent vaccinees. Such information will be crucial for regulatory decision-making about product registration and/or licensure.

The severe threat to public health posed by the COVID-19 pandemic requires sensitive balancing of the interests of participants in trials of vaccines against COVID-19 versus the need to conduct valuable and urgently needed research into vaccines against COVID-19, in the interest of public health. The policy brief developed by Working Group attempts to strike this delicate balance.

\section{Jerome Amir Singh $\left(1{ }^{1,2} \bowtie\right.$, Sonali Kochhar ${ }^{3,4}$,}

Jonathan Wolff ${ }^{5}$ and The WHO

ACT-Accelerator Ethics \& Governance Working Group*

${ }^{1}$ Howard College School of Law, University of KwaZulu-Natal, Durban, South Africa. ${ }^{2}$ Dalla Lana School of Public Health, University of Toronto, Toronto, Ontario, Canada. ${ }^{3}$ Department of Global Health, University of Washington, Seattle, WA, USA. ${ }^{4}$ Global Healthcare Consulting, Delhi, India. ${ }^{5}$ Blavatnik School of Government, University of Oxford, Oxford, UK. ${ }^{*} A$ list of authors and their affiliations appears at the end of the paper. $\bigotimes_{e-m a i l: s i n g h j 9 @ u k z n . a c . z a}$

Published online: 16 March 2021 https://doi.org/10.1038/s41591-021-01299-5

References

1. World Health Organization. https://www.who.int/publications/ $\mathrm{m} /$ item/draft-landscape-of-covid-19-candidate-vaccines (accessed 2 March 2021).

2. Medicines and Healthcare products Regulatory Agency. https://www.gov.uk/government/news/uk-medicinesregulator-gives-approval-for-first-uk-covid-19-vaccine (2 December 2020).
3. U.S. Food \& Drug Administration. https://www.fda.gov/newsevents/press-announcements/fda-takes-key-actionfight-against-covid-19-issuing-emergency-use-authorizationfirst-covid-19 (11 December 2020).

4. European Medicines Agency. https://www.ema.europa.eu/en/ news/ema-recommends-first-covid-19-vaccine-authorisation-eu (21 December 2020)

5. World Health Organization. https://www.who.int/news/item/ 31-12-2020-who-issues-its-first-emergency-use-validationfor-a-covid-19-vaccine-and-emphasizes-need-forequitable-global-access (31 December 2020).

6. World Health Organization. https://www.who.int/news/item/ 15-02-2021-who-lists-two-additional-covid-19-vaccines-foremergency-use-and-covax-roll-out\#: :text=Today $\% 20 \mathrm{WHO} \% 20$ listed $\% 20$ two $\% 20$ versions, the $\% 20$ Serum $\% 20$ Institute $\% 20$ of $\% 20$ India (15 February 2021).

7. World Medical Association. https://www.wma.net/policies-post/ wma-declaration-of-helsinki-ethical-principles-formedical-research-involving-human-subjects/ (2013).

8. Council for International Organizations of Medical Sciences. https://cioms.ch/publications/product/internationalethical-guidelines-for-health-related-research-involvinghumans/ (2016).

9. Rid, A., Saxena, A., Baqui, A. H. \& Bhan, A. et al. Vaccine 32, 4708-4712 (2014)

10. World Health Organization. https://apps.who.int/iris/ bitstream/handle/10665/337940/WHO-2019-nCoVPolicy_Brief-EUD_placebo-controlled_vaccine_trials-2020.1-eng. pdf? sequence $=1$ \&isAllowed $=y$ (18 December 2020).

\section{Acknowledgements}

The policy brief reported in this work was supported by the grant WHO COVID-19 SPRP R\&D, funded by the Ministry of Health of Germany. K. Littler, A. Reis (WHO Health Ethics \& Governance Unit), J. Hombach and A. Wilder-Smith (WHO Strategic Advisory Group of Experts on Immunization Secretariat) provided support for the WHO Secretariat. Contributions from G. Enwere and C. Rodriguez (WHO Prequalification/Vaccines \& Immunization Devices Assessment Unit), M.-C. Bouësseau (WHO Department of Integrated Health Services) and P. Hummel (WHO Consultant) are acknowledged.

Competing interests

The authors declare no competing interests.

\section{The WHO ACT-Accelerator Ethics \& Governance Working Group}

Caesar Atuire ${ }^{6}$, Anant Bhan7, Ezekiel Emanuel ${ }^{8}$, Ruth Faden ${ }^{9}$, Prakash Ghimire ${ }^{10}$,

Dirceu Greco"1 , Calvin $\mathrm{Ho}^{12}$, Suerie Moon ${ }^{13}$, Ehsan Shamsi-Gooshki ${ }^{14}$, Aissatou Touré ${ }^{15}$, Beatriz Thomé ${ }^{16}$, Maxwell J. Smith ${ }^{17}$ and Ross E. G. Upshur ${ }^{18}$

${ }^{6}$ University of Ghana, Accra, Ghana. ${ }^{7}$ Yenepoya University, Mangalore, India. ${ }^{8}$ University of Pennsylvania, Philadelphia, PA, USA. ${ }^{9}$ Johns Hopkins University, Baltimore, MD, USA. ${ }^{10}$ Tribhuvan University, Kirtipur, Nepal.

${ }^{11} \mathrm{Federal}$ University of Minas Gerais, Belo Horizonte, Brazil. ${ }^{12}$ University of Hong Kong, Hong Kong, China. ${ }^{13}$ Graduate Institute, Geneva, Switzerland. ${ }^{14}$ Tehran University of Medical Sciences, Tehran, Iran. ${ }^{15}$ Institut Pasteur, Dakar, Senegal. ${ }^{16}$ Federal University of Sao Paolo, Sao Paolo, Brazil. ${ }^{17}$ Western University, London, Ontario, Canada. ${ }^{18}$ University of Toronto, Toronto, Ontario, Canada. 